\title{
Analysis of Single Server Fixed Batch Service Queueing System under Multiple Vacations with Gated Service
}

\author{
G. Ayyappan \\ Professor \\ Pondicherry Engineering \\ College \\ Pondicherry
}

\author{
G. Devipriya \\ Assistant Professor \\ Sri Ganesh College of \\ Engineering \& Technology \\ Pondicherry
}

\author{
A. Muthu Ganapathi \\ Subramanian \\ Associate Professor \\ Kanchi Mamunivar Centre for \\ Post Graduate Studies \\ Pondicherry
}

\begin{abstract}
Consider a single server fixed batch service queueing system under multiple vacation with gated service in which the arrival rate $\lambda$ follows a Poisson process and the service time follows an exponential distribution with parameter $\mu$. Assume that the system initially contain $\mathrm{k}$ customers when the server enters into the system and starts the service immediately in batch of size $\mathrm{k}$. After completion of a service, if he finds less than $\mathrm{k}$ customers in the queue, then the server goes for a multiple vacation of length $\alpha$. If there are more than $\mathrm{k}$ customers in the queue then the first $\mathrm{k}$ customers will be selected from the queue and service will be given as a batch. Gated type service policy is adopted in this model that is once the server starts service for a batch of $\mathrm{k}$ customers, no customers will be allowed to enter into the queue. Every time a service is finished, and there are less than $\mathrm{k}$ customers in the queue, the server leaves for a vacation of length $\alpha$. This model is completely solved by constructing the generating function and Rouche's theorem is applied and we have derived the closed form solutions for probability of number of customers in the queue during the server busy and in vacation. Further we are providing the closed form solutions for mean number of customers in the queue, variance and various system performance measures of the system. Numerical studies have been done for analysis of system measures for various values of $\lambda, \mu, \alpha$ and $\mathrm{k}$.
\end{abstract}

\section{Keywords}

Single Server, Batch Service, gated service, multiple vacation, Steady state distribution.

\section{INTRODUCTION}

Batch service queues have numerous applications to traffic, transportation, production, and manufacturing systems. Bailey[2] obtained the transform solution to the fixed-size batch service queue with Poisson arrivals that was the first study on batch service queues. Miller[16] have studied the batch arrival batch service queues and Jaiswal[13] considered batch service queues in which service size is random. Ayyappan et al [1] have studied the single server batch service Queueing system under multiple vacation with Catastrophe. Neuts[17] have proposed the "general bulk service rule" in which service initiates only when a certain number of customers in the queue is available. Neuts general bulk service rule was extended by Borthakur and Medhi[3]. Studies on waiting time in a batch service queue were also rendered by Downton[10], Cohen[6], Medhi[15] and Powell[18]. Fakinos [10] derived the relation between limiting queue size distributions at arrival and departure epochs. Briere and Chaudhry[4], Grassmann and Chaudhry[12], and Kambo and Chaudhry[14] used numerical approaches to obtain the performance measures. Numerical methods were found to be effective especially for batch service queues, because the transform solution of the queue length (number of customers in the system including those in service) in batch service systems contains some unknown values. Chaudhry and Templeton[5] give more extensive study on batch arrival/service queues.

Vacation queues have been extensively studied by many researchers. Comprehensive surveys can be found in Doshi[8] and Takagi[19].

For batch service queues with vacations, there have been a few related works. Dhas[6] considered Markovian batch service systems and obtained the queue length distributions by matrix-geometric methods. Dshalalow and Yellen[9] considered a non-exhaustive batch service system with multiple vacations in which the server starts a multiple vacation whenever the queue drops below a level $r$ and resumes service at the end of a vacation segment when the queue accumulates to at least $r$. They called such a system (r, R)-quorum system.

In this paper we are going to concentrate on a special batch service queue called the fixed size batch service queue under multiple vacations with gated service. The model under consideration is described in Section 2. In Section 3, analyze the model by deriving the system steady state equations. Using these equations, the probability generating functions are obtained and steady state probabilities are obtained. A numerical study is carried out in Section 6 to test the effect of the system performance measure discussed in Section 5 . We are providing the closed form solutions for system performance measures. Numerical studies have been done for system performance measures for various values of $\lambda, \mu, \alpha$, and $\mathrm{k}$.

\section{MODEL DESCRIPATION AND}

\section{ANALYSIS}

Consider a single server fixed batch service queueing system under multiple vacation with gated service in which the arrival rate $\lambda$ follows a Poisson process and the service time follows an exponential distribution with parameter $\mu$. Assume that the system initially contain $\mathrm{k}$ customers when the server enters into the system and starts the service immediately in a batch of size $\mathrm{k}$. After service completion, If there are more than $\mathrm{k}$ customers in the queue then the first $\mathrm{k}$ customers will be selected from the queue and service will be given as a batch. Gated type service policy is adopted is this model that is once the server starts service for a batch of $\mathrm{k}$ customers, no customers will be allowed to enter into the queue. After completion of a service, if he finds less than $\mathrm{k}$ customers in the queue, then the server goes for a multiple vacation of 
length $\alpha$. Upon his return from the vacation if he finds less than $\mathrm{k}$ customers in the queue, he immediately leaves for another vacation and so on until he finally finds $\mathrm{k}$ or more customers waiting in the queue. Let $\langle\mathrm{N}(\mathrm{t}), \mathrm{C}(\mathrm{t})\rangle$ be a random process where $\mathrm{N}(\mathrm{t})$ be the random variable which represents the number of customers in queue at time $t$ and $C(t)$ be the random variable which represents the server status (busy/vacation) at time t.

We define

$P_{n, 1}(t)$ - Probability that the server is busy if there are $n$ customers in the queue at time $t$.

$P_{n, 2}(t)$ - Probability that the server is in vacation if there are $n$ customers in the queue at time $t$

The Chapman- Kolmogorov equations are

$$
\begin{aligned}
& P_{0,1}^{\prime}(t)=-\mu P_{0,1}(t)+\mu P_{k, 1}(t)+\alpha \mathrm{P}_{k, 2}(\mathrm{t}) \\
& P_{n, 1}^{\prime}(t)=-\mu P_{n, 1}(t)+\mu P_{n+k, 1}(t)+\alpha \mathrm{P}_{n+k, 2}(\mathrm{t})
\end{aligned}
$$

for $\mathrm{n}=1,2$..

$$
\begin{aligned}
& P_{0,2}^{\prime}(t)=-\lambda P_{0,2}(t)+\mu P_{0,1}(t) \\
& P_{n, 2}^{\prime}(t)=-\lambda P_{n, 2}(t)+\lambda P_{n-1,2}(t)+\mu P_{n, 1}(t)
\end{aligned}
$$

for $\mathrm{n}=1,2 \ldots$

$$
P_{n, 2}^{\prime}(t)=-(\lambda+\alpha) P_{n, 2}(t)+\lambda P_{n-1,2}(t) \text { for } \mathrm{n} \geq \mathrm{k}
$$

\section{EVALUATION OF STEADY STATE PROBABILITY}

In this section, we are finding the closed form solutions for number of customers in the queue when the server is busy or in vacation by using Generating functions.

When steady state prevails, the equations (1) to (5) becomes

$\mu P_{0,1}=\mu P_{k, 1}+\alpha \mathrm{P}_{k, 2}$

$\mu P_{n, 1}=\mu P_{n+k, 1}+\alpha \mathrm{P}_{n+k, 2}$ for $\mathrm{n}=1,2,3, \ldots$

$\lambda P_{0,2}=\mu P_{0,1}$

$\lambda P_{n, 2}=\lambda P_{n-1,2}+\mu P_{n, 1}$ for $\mathrm{n}=1,2,3, \ldots, \mathrm{k}-1$

$(\lambda+\alpha) P_{n, 2}=\lambda P_{n-1,2}$ for $\mathrm{n} \geq \mathrm{k}$

Generating functions for the number of customers in the queue when the server is busy or in vacation are defined as

$$
G(z)=\sum_{n=0}^{\infty} P_{n, 1} z^{n} \text { and } H(z)=\sum_{n=0}^{\infty} P_{n, 2} z^{n}
$$

Adding equations (6) and (7) after Multiplying by $1, \mathrm{z}^{\mathrm{n}}(\mathrm{n}=$ 0 to $\infty$ ) respectively

$$
G(z) \mu\left(1-z^{k}\right)+\alpha H(z)=\mu \sum_{n=0}^{k-1} P_{n, 1} z^{n}+\alpha \sum_{n=0}^{k-1} P_{n, 2} z^{n}
$$

Adding equations (8), (9) and (10) after Multiplying by $1, \mathrm{z}^{\mathrm{n}}$ and $\mathrm{z}^{\mathrm{n}}(\mathrm{n}=1,2,3, \ldots)$ respectively

$$
\begin{aligned}
& H(z)[\alpha+\lambda(1-z)]=\mu \sum_{n=0}^{k-1} P_{n, 1} z^{n}+\alpha \sum_{n=0}^{k-1} P_{n, 2} z^{n} \\
& H(z)=\frac{\mu \sum_{n=0}^{k-1} P_{n, 1} z^{n}+\alpha \sum_{n=0}^{k-1} P_{n, 2} z^{n}}{\alpha+\lambda(1-z)}
\end{aligned}
$$

Equation (13) represents the probability generating function for number of customers in the queue when the server is in vacation.
From (11) and (13)

$$
G(z)=\frac{\lambda(1-z) H(z)}{\mu\left(1-z^{k}\right)}
$$

From (13) and (14)

$$
G(z)=\frac{\left(\frac{\lambda}{\mu}\right)(1-z)\left(\mu \sum_{n=0}^{k-1} P_{n, 1} z^{n}+\alpha \sum_{n=0}^{k-1} P_{n, 2} z^{n}\right)}{\lambda z^{k+1}-(\lambda+\alpha) z^{k}-\lambda z+(\lambda+\alpha)}
$$

Equation (15) represents the probability generating function for number of customers in the queue when the server is busy. The generating function $\mathrm{G}(\mathrm{z})$ has the property that it must converge inside the unit circle $|z|<1$. We notice that the expression in the denominator of $\mathrm{G}(\mathrm{z})$, $\lambda z^{k+1}-(\lambda+\alpha) z^{k}-\lambda z+(\lambda+\alpha)$ has $\mathrm{k}+1$ zeros. By Rouche's theorem, we notice that $\mathrm{k}$ zeros of this expression lies inside the circle $|z|<1$ and must coincide with $\mathrm{k}$ zeros of numerator of $\mathrm{G}(\mathrm{z})$ and one zero lies outside the circle $|z|<1$. Let $\mathrm{z}_{0}$ be a zero which lies outside the circle $|z|<1$

As $\mathrm{G}(\mathrm{z})$ converges, $\mathrm{k}$ zeros of numerator and denominator equation (13) will be cancelled, then

$$
G(z)=\frac{A}{\mu\left(z-z_{0}\right)}
$$

When $\mathrm{z}=1$, from the equation (14)

$$
G(1)=\frac{\lambda}{k \mu} H(1)
$$

The normalized condition is $\mathrm{G}(1)+\mathrm{H}(1)=1$

From (17) and (18)

$$
H(1)=\left(1+\frac{\lambda}{k \mu}\right)^{-1}
$$

When $\mathrm{z}=1, G(1)=\frac{A}{\mu\left(1-z_{0}\right)}$

From the equations (17), (19) and (20)

$$
A=\frac{\lambda}{k \mu}\left(1+\frac{\lambda}{k \mu}\right)^{-1}\left(1-z_{0}\right)
$$

From (16) and (21)

$$
G(z)=\frac{\lambda / k \mu^{\left(z_{0}-1\right)}}{1+\lambda / k \mu} \sum_{n=0}^{\infty} \frac{z^{n}}{z_{0}^{n+1}}
$$

Comparing the coefficient of $\mathrm{z}^{\mathrm{n}}$ on both sides of the equation (22)

$$
P_{n, 1}=\frac{\lambda / k \mu^{\left(z_{0}-1\right)}}{1+\lambda / k \mu}\left(\frac{1}{z_{0}^{n+1}}\right) \text { for } \mathrm{n}=0,1,2, \ldots
$$

Using equations (7), (8) and (9) and apply recursively for $\mathrm{n}=$ $1,2,3, \ldots, \mathrm{k}-1$

$$
P_{n, 2}=\frac{\mu}{\lambda} \sum_{t=0}^{n} P_{t, 1} \text { for } \mathrm{n}=0,1,2, \ldots, \mathrm{k}-1
$$




$$
P_{n, 2}=\left(\frac{\lambda}{\lambda+\alpha}\right)^{n-k+1} P_{k-1,2} \text { for } \mathrm{n} \geq \mathrm{k}
$$

Equations (23),(24) and (25) represents closed form solutions of the steady state probabilities for number of customers in the queue when the server is busy or in vacation.

\section{STABILITY CONDITION}

The necessary and sufficient condition for the system to the stable is $\frac{\lambda}{k \mu}<1$.

\section{SYSTEMPERFORMANCE MEASURES}

In this section, list out some important performance measures along with their formulas. These measures are used to bring out the qualitative behaviour of the queueing model under study. Numerical study has been dealt in very large scale to study the following measures.

$$
\begin{aligned}
& \text { 1. } P_{n, 1}=\frac{\lambda / k \mu{ }_{0}^{\left(z_{0}-1\right)}}{1+\lambda / k \mu}\left(\frac{1}{z_{0}^{n+1}}\right) \text { for } \mathrm{n}=0,1,2, \ldots \\
& \text { 2. } P_{n, 2}=\frac{\mu}{\lambda} \sum_{t=0}^{n} P_{t, 1} \text { for } \mathrm{n}=0,1,2, \ldots, \mathrm{k}-1 \\
& \text { 3. } \quad P_{n, 2}=\left(\frac{\lambda}{\lambda+\alpha}\right)^{n-k+1} P_{k-1,2} \text { for } \mathrm{n} \geq \mathrm{k} \\
& \text { 4. } L_{q}=\sum_{n=0}^{\infty} n\left(P_{n, 1}+P_{n, 2}\right) \\
& \text { 5. } \quad V(x)=\left(\sum_{n=0}^{\infty} n^{2}\left(P_{n, 1}+P_{n, 2}\right)\right)-\left(L_{q}\right)^{2}
\end{aligned}
$$

\section{NUMERICAL STUDIES}

The values of parameters $\lambda, \mu, \alpha, \mathrm{k}$ are chosen so that they satisfy the stability condition discussed in section 4 .

The system performance measures of this model have been done and expressed in the form of tables for various values $\lambda$, $\mu, \alpha$ and $\mathrm{k}$

Tables 3, 6, 9 and 12 show the impact of arrival rate $\lambda$ and $\mathrm{k}$ over Mean number of customers in the queue. Further we infer the following

- Mean number of customers in the queue increases as arrival rate $\lambda$ increases.

- $\mathrm{P}_{1}$ increases and $\mathrm{P}_{2}$ decreases as arrival rate $\lambda$ increases.

Tables $1,4,7$ and 10 show the steady probabilities distribution when server is busy for various values of $\lambda$ and different batch size $\mathrm{k}$.

Tables 2,5,8and11 show the steady state probabilities distribution when the server is in vacation for various values of $\lambda$ and different batch size $\mathrm{k}$.
Table 1: Steady state Probabilities distribution for various values of $\lambda$ and $\mu=10, \alpha=5$ when the server is busy and batch size is $\mathbf{k}=\mathbf{2}$

\begin{tabular}{|c|l|l|l|l|l|l|}
\hline$\lambda$ & P01 & P11 & P21 & P31 & P41 & P51 \\
\hline 1.0 & 0.0397 & 0.0066 & 0.0011 & 0.0002 & 0.0000 & 0.0000 \\
\hline 2.0 & 0.0649 & 0.0186 & 0.0053 & 0.0015 & 0.0004 & 0.0001 \\
\hline 3.0 & 0.0815 & 0.0306 & 0.0115 & 0.0043 & 0.0016 & 0.0006 \\
\hline 4.0 & 0.0926 & 0.0412 & 0.0183 & 0.0081 & 0.0036 & 0.0016 \\
\hline 5.0 & 0.1000 & 0.0500 & 0.0250 & 0.0125 & 0.0063 & 0.0031 \\
\hline 6.0 & 0.1049 & 0.0572 & 0.0312 & 0.0170 & 0.0093 & 0.0051 \\
\hline 7.0 & 0.1080 & 0.0630 & 0.0368 & 0.0214 & 0.0125 & 0.0073 \\
\hline 8.0 & 0.1099 & 0.0676 & 0.0416 & 0.0256 & 0.0158 & 0.0097 \\
\hline 9.0 & 0.1108 & 0.0713 & 0.0458 & 0.0294 & 0.0189 & 0.0122 \\
\hline
\end{tabular}

Table2:Steady state Probabilities distribution for various values of $\lambda$ and $\mu=10, \alpha=5$ when the server is in vacation and batch size is $K=2$

\begin{tabular}{|l|l|l|l|l|l|l|}
\hline$\lambda$ & P02 & P12 & P22 & P32 & P42 & P52 \\
\hline 1 & 0.3968 & 0.4630 & 0.0772 & 0.0129 & 0.0021 & 0.0004 \\
\hline 2 & 0.3247 & 0.4174 & 0.1193 & 0.0341 & 0.0097 & 0.0028 \\
\hline 3 & 0.2717 & 0.3736 & 0.1401 & 0.0525 & 0.0197 & 0.0074 \\
\hline 4 & 0.2315 & 0.3344 & 0.1486 & 0.0660 & 0.0294 & 0.0130 \\
\hline 5 & 0.2000 & 0.3000 & 0.1500 & 0.0750 & 0.0375 & 0.0188 \\
\hline 6 & 0.1748 & 0.2702 & 0.1474 & 0.0804 & 0.0438 & 0.0239 \\
\hline 7 & 0.1543 & 0.2443 & 0.1425 & 0.0831 & 0.0485 & 0.0283 \\
\hline 8 & 0.1374 & 0.2219 & 0.1365 & 0.0840 & 0.0517 & 0.0318 \\
\hline 9 & 0.1232 & 0.2023 & 0.1301 & 0.0836 & 0.0538 & 0.0346 \\
\hline
\end{tabular}

Table 3: Average number of customers in the queue and Variance for various values of $\lambda, \mu=10, \alpha=5$ and batch size is $\mathbf{K}=\mathbf{2}$

\begin{tabular}{|c|c|c|c|c|c|c|}
\hline$\lambda$ & $\mu$ & $\mathrm{A}$ & $\mathrm{P} 1$ & $\mathrm{P} 2$ & Mean & Variance \\
\hline 1 & 10 & 5 & 0.0476 & 0.9524 & 0.6762 & 0.4894 \\
\hline 2 & 10 & 5 & 0.0909 & 0.9091 & 0.8545 & 0.8079 \\
\hline 3 & 10 & 5 & 0.1304 & 0.8696 & 1.0348 & 1.2057 \\
\hline 4 & 10 & 5 & 0.1667 & 0.8333 & 1.2167 & 1.6831 \\
\hline 5 & 10 & 5 & 0.2000 & 0.8000 & 1.4000 & 2.2400 \\
\hline 6 & 10 & 5 & 0.2308 & 0.7692 & 1.5846 & 2.8767 \\
\hline 7 & 10 & 5 & 0.2593 & 0.7407 & 1.7704 & 3.5932 \\
\hline 8 & 10 & 5 & 0.2857 & 0.7143 & 1.9571 & 4.3896 \\
\hline 9 & 10 & 5 & 0.3103 & 0.6897 & 2.1448 & 5.2659 \\
\hline
\end{tabular}

Table 4: Steady state Probabilities distribution for various values of $\lambda$ and $\mu=10, \alpha=5$ when the server is busy and batch size is $K=3$

\begin{tabular}{|l|l|l|l|l|l|l|}
\hline$\lambda$ & $\mathrm{P}_{01}$ & $\mathrm{P}_{11}$ & $\mathrm{P}_{21}$ & $\mathrm{P}_{31}$ & $\mathrm{P}_{41}$ & $\mathrm{P}_{51}$ \\
\hline 1 & 0.0269 & 0.0045 & 0.0007 & 0.0001 & 0.0000 & 0.0000 \\
\hline 2 & 0.0446 & 0.0128 & 0.0036 & 0.0010 & 0.0003 & 0.0001 \\
\hline 3 & 0.0568 & 0.0213 & 0.0080 & 0.0030 & 0.0011 & 0.0004 \\
\hline 4 & 0.0654 & 0.0290 & 0.0129 & 0.0057 & 0.0026 & 0.0011 \\
\hline 5 & 0.0714 & 0.0357 & 0.0179 & 0.0089 & 0.0045 & 0.0022 \\
\hline 6 & 0.0758 & 0.0413 & 0.0225 & 0.0123 & 0.0067 & 0.0037 \\
\hline 7 & 0.0788 & 0.0460 & 0.0268 & 0.0156 & 0.0091 & 0.0053 \\
\hline 8 & 0.0810 & 0.0498 & 0.0307 & 0.0189 & 0.0116 & 0.0071 \\
\hline 9 & 0.0824 & 0.0530 & 0.0341 & 0.0219 & 0.0141 & 0.0090 \\
\hline
\end{tabular}


Table 5: Steady state Probabilities distribution for various values of $\lambda$ and $\mu=10, \alpha=5$ when the server is in vacation and batch size is $K=3$

\begin{tabular}{|l|l|l|l|l|l|l|}
\hline$\Lambda$ & $\mathrm{P}_{02}$ & $\mathrm{P}_{12}$ & $\mathrm{P}_{22}$ & $\mathrm{P}_{32}$ & $\mathrm{P}_{42}$ & $\mathrm{P}_{52}$ \\
\hline 1 & 0.2688 & 0.3136 & 0.3211 & 0.0535 & 0.0089 & 0.0015 \\
\hline 2 & 0.2232 & 0.2870 & 0.3052 & 0.0872 & 0.0249 & 0.0071 \\
\hline 3 & 0.1894 & 0.2604 & 0.2871 & 0.1076 & 0.0404 & 0.0151 \\
\hline 4 & 0.1634 & 0.2360 & 0.2683 & 0.1192 & 0.0530 & 0.0236 \\
\hline 5 & 0.1429 & 0.2143 & 0.2500 & 0.1250 & 0.0625 & 0.0313 \\
\hline 6 & 0.1263 & 0.1951 & 0.2327 & 0.1269 & 0.0692 & 0.0378 \\
\hline 7 & 0.1126 & 0.1783 & 0.2166 & 0.1264 & 0.0737 & 0.0430 \\
\hline 8 & 0.1012 & 0.1635 & 0.2018 & 0.1242 & 0.0764 & 0.0470 \\
\hline 9 & 0.0916 & 0.1504 & 0.1883 & 0.1210 & 0.0778 & 0.0500 \\
\hline
\end{tabular}

Table 6: Average number of customers in the queue and Variance for various values of $\lambda$ and $\mu=10, \alpha=5$ and batch size is $\mathrm{K}=\mathbf{3}$

\begin{tabular}{|l|l|l|l|l|l|l|}
\hline$\lambda$ & $\mu$ & $\alpha$ & $\mathrm{P}_{1}$ & $\mathrm{P} 2$ & Mean & Variance \\
\hline 1 & 10 & 5 & 0.0323 & 0.9677 & 1.1677 & 0.9164 \\
\hline 2 & 10 & 5 & 0.0625 & 0.9375 & 1.3375 & 1.2436 \\
\hline 3 & 10 & 5 & 0.0909 & 0.9091 & 1.5091 & 1.6487 \\
\hline 4 & 10 & 5 & 0.1176 & 0.8824 & 1.6824 & 2.1320 \\
\hline 5 & 10 & 5 & 0.1429 & 0.8571 & 1.8571 & 2.6939 \\
\hline 6 & 10 & 5 & 0.1667 & 0.8333 & 2.0333 & 3.3344 \\
\hline 7 & 10 & 5 & 0.1892 & 0.8108 & 2.2108 & 4.0539 \\
\hline 8 & 10 & 5 & 0.2105 & 0.7895 & 2.3895 & 4.8525 \\
\hline 9 & 10 & 5 & 0.2308 & 0.7692 & 2.5692 & 5.7303 \\
\hline
\end{tabular}

Table 7: Steady state Probabilities distribution for various values of $\lambda$ and $\mu=10, \alpha=5$ when the server is busy and batch size is $K=4$

\begin{tabular}{|l|l|l|l|l|l|l|}
\hline$\lambda$ & $\mathrm{P}_{01}$ & $\mathrm{P}_{11}$ & $\mathrm{P}_{21}$ & $\mathrm{P}_{31}$ & $\mathrm{P}_{41}$ & $\mathrm{P}_{51}$ \\
\hline 1 & 0.0203 & 0.0034 & 0.0006 & 0.0001 & 0.0000 & 0.0000 \\
\hline 2 & 0.0340 & 0.0097 & 0.0028 & 0.0008 & 0.0002 & 0.0001 \\
\hline 3 & 0.0436 & 0.0164 & 0.0061 & 0.0023 & 0.0009 & 0.0003 \\
\hline 4 & 0.0505 & 0.0224 & 0.0100 & 0.0044 & 0.0020 & 0.0009 \\
\hline 5 & 0.0556 & 0.0278 & 0.0139 & 0.0069 & 0.0035 & 0.0017 \\
\hline 6 & 0.0593 & 0.0323 & 0.0176 & 0.0096 & 0.0052 & 0.0029 \\
\hline 7 & 0.0621 & 0.0362 & 0.0211 & 0.0123 & 0.0072 & 0.0042 \\
\hline 8 & 0.0641 & 0.0394 & 0.0243 & 0.0149 & 0.0092 & 0.0057 \\
\hline 9 & 0.0656 & 0.0422 & 0.0271 & 0.0174 & 0.0112 & 0.0072 \\
\hline
\end{tabular}

Table 8: Steady state Probabilities distribution for various values of $\lambda$ and $\mu=10, \alpha=5$ when the server is in vacation and batch size is $K=4$

\begin{tabular}{|l|l|l|l|l|l|l|}
\hline$\Lambda$ & $\mathrm{P}_{02}$ & $\mathrm{P}_{12}$ & $\mathrm{P}_{22}$ & $\mathrm{P}_{32}$ & $\mathrm{P}_{42}$ & $\mathrm{P}_{52}$ \\
\hline 1 & 0.2033 & 0.2371 & 0.2428 & 0.2437 & 0.0406 & 0.0068 \\
\hline 2 & 0.1701 & 0.2187 & 0.2325 & 0.2365 & 0.0676 & 0.0193 \\
\hline 3 & 0.1453 & 0.1999 & 0.2203 & 0.2280 & 0.0855 & 0.0321 \\
\hline 4 & 0.1263 & 0.1824 & 0.2073 & 0.2184 & 0.0971 & 0.0431 \\
\hline 5 & 0.1111 & 0.1667 & 0.1944 & 0.2083 & 0.1042 & 0.0521 \\
\hline 6 & 0.0988 & 0.1527 & 0.1821 & 0.1981 & 0.1081 & 0.0590 \\
\hline 7 & 0.0887 & 0.1404 & 0.1705 & 0.1881 & 0.1097 & 0.0640 \\
\hline 8 & 0.0801 & 0.1294 & 0.1598 & 0.1785 & 0.1098 & 0.0676 \\
\hline 9 & 0.0729 & 0.1197 & 0.1499 & 0.1692 & 0.1088 & 0.0699 \\
\hline
\end{tabular}

Table 9: Average number of customers in the queue and Variance for various values of $\lambda$ and $\mu=10, \alpha=5$ and batch size is $K=4$

\begin{tabular}{|l|l|l|l|l|l|l|}
\hline$\lambda$ & $\mu$ & $\alpha$ & $\mathrm{P}_{1}$ & $\mathrm{P} 2$ & Mean & Variance \\
\hline 1 & 10 & 5 & 0.0244 & 0.9756 & 1.6634 & 1.5131 \\
\hline 2 & 10 & 5 & 0.0476 & 0.9524 & 1.8286 & 1.8525 \\
\hline 3 & 10 & 5 & 0.0698 & 0.9302 & 1.9953 & 2.2688 \\
\hline 4 & 10 & 5 & 0.0909 & 0.9091 & 2.1636 & 2.7623 \\
\hline 5 & 10 & 5 & 0.1111 & 0.8889 & 2.3333 & 3.3333 \\
\hline 6 & 10 & 5 & 0.1304 & 0.8696 & 2.5043 & 3.9822 \\
\hline 7 & 10 & 5 & 0.1489 & 0.8511 & 2.6766 & 4.7090 \\
\hline 8 & 10 & 5 & 0.1667 & 0.8333 & 2.8500 & 5.5142 \\
\hline 9 & 10 & 5 & 0.1837 & 0.8163 & 3.0245 & 6.3978 \\
\hline
\end{tabular}

Table 10: Steady state Probabilities distribution for various values of $\lambda$ and $\mu=10, \alpha=5$ when the server is busy and batch size is $K=5$

\begin{tabular}{|l|l|l|l|l|l|l|}
\hline$\lambda$ & $\mathrm{P}_{01}$ & $\mathrm{P}_{11}$ & $\mathrm{P}_{21}$ & $\mathrm{P}_{31}$ & $\mathrm{P}_{41}$ & $\mathrm{P}_{51}$ \\
\hline 1 & 0.0163 & 0.0027 & 0.0005 & 0.0001 & 0.0000 & 0.0000 \\
\hline 2 & 0.0275 & 0.0078 & 0.0022 & 0.0006 & 0.0002 & 0.0001 \\
\hline 3 & 0.0354 & 0.0133 & 0.0050 & 0.0019 & 0.0007 & 0.0003 \\
\hline 4 & 0.0412 & 0.0183 & 0.0081 & 0.0036 & 0.0016 & 0.0007 \\
\hline 5 & 0.0455 & 0.0227 & 0.0114 & 0.0057 & 0.0028 & 0.0014 \\
\hline 6 & 0.0487 & 0.0266 & 0.0145 & 0.0079 & 0.0043 & 0.0024 \\
\hline 7 & 0.0512 & 0.0298 & 0.0174 & 0.0102 & 0.0059 & 0.0035 \\
\hline 8 & 0.0531 & 0.0326 & 0.0201 & 0.0124 & 0.0076 & 0.0047 \\
\hline 9 & 0.0545 & 0.0350 & 0.0225 & 0.0145 & 0.0093 & 0.0060 \\
\hline
\end{tabular}

Table 11: Steady state Probabilities distribution for various values of $\lambda$ and $\mu=10, \alpha=5$ when the server is in vacation and batch size is $K=5$

\begin{tabular}{|l|l|l|l|l|l|l|}
\hline$\lambda$ & P02 & P12 & P22 & P32 & P42 & P52 \\
\hline 1 & 0.1634 & 0.1906 & 0.1952 & 0.1959 & 0.1961 & 0.0327 \\
\hline 2 & 0.1374 & 0.1766 & 0.1878 & 0.1910 & 0.1919 & 0.0548 \\
\hline 3 & 0.1179 & 0.1621 & 0.1787 & 0.1849 & 0.1873 & 0.0702 \\
\hline 4 & 0.1029 & 0.1486 & 0.1689 & 0.1780 & 0.1820 & 0.0809 \\
\hline 5 & 0.0909 & 0.1364 & 0.1591 & 0.1705 & 0.1761 & 0.0881 \\
\hline 6 & 0.0812 & 0.1254 & 0.1496 & 0.1628 & 0.1699 & 0.0927 \\
\hline 7 & 0.0731 & 0.1157 & 0.1406 & 0.1551 & 0.1636 & 0.0954 \\
\hline 8 & 0.0663 & 0.1071 & 0.1322 & 0.1477 & 0.1572 & 0.0967 \\
\hline 9 & 0.0605 & 0.0994 & 0.1245 & 0.1405 & 0.1509 & 0.0970 \\
\hline
\end{tabular}

Table 12: Average number of customers in the queue and Variance for various values of $\lambda$ and $\mu=10, \alpha=5$ and batch size is $K=5$

\begin{tabular}{|l|l|l|l|l|l|l|}
\hline$\lambda$ & $\mu$ & $\alpha$ & $\mathrm{P}_{1}$ & $\mathrm{P} 2$ & Mean & Variance \\
\hline 1 & 10 & 5 & 0.0196 & 0.9804 & 2.1608 & 2.2777 \\
\hline 2 & 10 & 5 & 0.0385 & 0.9615 & 2.3231 & 2.6310 \\
\hline 3 & 10 & 5 & 0.0566 & 0.9434 & 2.4868 & 3.0604 \\
\hline 4 & 10 & 5 & 0.0741 & 0.9259 & 2.6519 & 3.5662 \\
\hline 5 & 10 & 5 & 0.0909 & 0.9091 & 2.8182 & 4.1488 \\
\hline 6 & 10 & 5 & 0.1071 & 0.8929 & 2.9857 & 4.8084 \\
\hline 7 & 10 & 5 & 0.1228 & 0.8772 & 3.1544 & 5.5453 \\
\hline 8 & 10 & 5 & 0.1379 & 0.8621 & 3.3241 & 6.3598 \\
\hline 9 & 10 & 5 & 0.1525 & 0.8475 & 3.4949 & 7.2520 \\
\hline
\end{tabular}


Table 13: Steady state Probabilities distribution for various values of $\lambda$ and $\mu=10, \alpha=5$ when the server is busy and batch size is $K=6$

\begin{tabular}{|l|l|l|l|l|l|l|}
\hline$\lambda$ & $\mathrm{P}_{01}$ & $\mathrm{P}_{11}$ & $\mathrm{P}_{21}$ & $\mathrm{P}_{31}$ & $\mathrm{P}_{41}$ & $\mathrm{P}_{51}$ \\
\hline 1 & 0.0137 & 0.0023 & 0.0004 & 0.0001 & 0.0000 & 0.0000 \\
\hline 2 & 0.0230 & 0.0066 & 0.0019 & 0.0005 & 0.0002 & 0.0000 \\
\hline 3 & 0.0298 & 0.0112 & 0.0042 & 0.0016 & 0.0006 & 0.0002 \\
\hline 4 & 0.0347 & 0.0154 & 0.0069 & 0.0030 & 0.0014 & 0.0006 \\
\hline 5 & 0.0385 & 0.0192 & 0.0096 & 0.0048 & 0.0024 & 0.0012 \\
\hline 6 & 0.0413 & 0.0225 & 0.0123 & 0.0067 & 0.0037 & 0.0020 \\
\hline 7 & 0.0435 & 0.0254 & 0.0148 & 0.0086 & 0.0050 & 0.0029 \\
\hline 8 & 0.0452 & 0.0278 & 0.0171 & 0.0105 & 0.0065 & 0.0040 \\
\hline 9 & 0.0466 & 0.0299 & 0.0193 & 0.0124 & 0.0080 & 0.0051 \\
\hline
\end{tabular}

Table 14: Steady state Probabilities distribution for various values of $\lambda$ and $\mu=10, \alpha=5$ when the server is in vacation and batch size is $K=6$

\begin{tabular}{|l|l|l|l|l|l|l|}
\hline$\Lambda$ & $\mathrm{P}_{02}$ & $\mathrm{P}_{12}$ & $\mathrm{P}_{22}$ & $\mathrm{P}_{32}$ & $\mathrm{P}_{42}$ & $\mathrm{P}_{52}$ \\
\hline 1 & 0.1366 & 0.1594 & 0.1632 & 0.1638 & 0.1639 & 0.1639 \\
\hline 2 & 0.1152 & 0.1481 & 0.1575 & 0.1602 & 0.1610 & 0.1612 \\
\hline 3 & 0.0992 & 0.1364 & 0.1504 & 0.1556 & 0.1576 & 0.1583 \\
\hline 4 & 0.0868 & 0.1254 & 0.1425 & 0.1502 & 0.1535 & 0.1550 \\
\hline 5 & 0.0769 & 0.1154 & 0.1346 & 0.1442 & 0.1490 & 0.1514 \\
\hline 6 & 0.0689 & 0.1064 & 0.1269 & 0.1381 & 0.1442 & 0.1475 \\
\hline 7 & 0.0622 & 0.0985 & 0.1196 & 0.1320 & 0.1392 & 0.1434 \\
\hline 8 & 0.0566 & 0.0914 & 0.1128 & 0.1260 & 0.1341 & 0.1391 \\
\hline 9 & 0.0518 & 0.0850 & 0.1064 & 0.1202 & 0.1290 & 0.1347 \\
\hline
\end{tabular}

Table 15: Average number of customers in the queue and Variance for various values of $\lambda$ and $\mu=10, \alpha=5$ and batch size is $K=6$

\begin{tabular}{|l|l|l|l|l|l|l|}
\hline$\lambda$ & $\mu$ & $\mathrm{A}$ & $\mathrm{P}_{1}$ & $\mathrm{P} 2$ & Mean & Variance \\
\hline 1 & 10 & 5 & 0.0164 & 0.9836 & 2.6590 & 3.2096 \\
\hline 2 & 10 & 5 & 0.0323 & 0.9677 & 2.8194 & 3.5777 \\
\hline 3 & 10 & 5 & 0.0476 & 0.9524 & 2.9810 & 4.0212 \\
\hline 4 & 10 & 5 & 0.0625 & 0.9375 & 3.1438 & 4.5406 \\
\hline 5 & 10 & 5 & 0.0769 & 0.9231 & 3.3077 & 5.1361 \\
\hline 6 & 10 & 5 & 0.0909 & 0.9091 & 3.4727 & 5.8080 \\
\hline 7 & 10 & 5 & 0.1045 & 0.8955 & 3.6388 & 6.5567 \\
\hline 8 & 10 & 5 & 0.1176 & 0.8824 & 3.8059 & 7.3823 \\
\hline 9 & 10 & 5 & 0.1304 & 0.8696 & 3.9739 & 8.2851 \\
\hline
\end{tabular}

\section{CONCLUSION}

The Numerical studies show the changes in the system due to impact of batch size, vacation rate and arrival rate. The mean number of customers in the queue increases as batch size and arrival rate increase. This research work can be extended further by introducing various concepts like vacation interruptions, breakdown and repair etc

\section{REFERENCES}

[1] G.Ayyappan,,G.Devipriya and A. Muthu Ganapthi Subramanian., 2013, Analysis of Single Server Fixed Batch Service Queueing System under Multiple Vacation with Catastrophe, at Mathematical Theory and Modeling Vol.3, No.11,
[2] Bailey, N.T.J., 1954, On queueing process with bulk service, Journal of Royal Statistical Society, B16,80-97.

[3] Borthakur, A. and Medhi, J., 1973, A queueing system with arrival and service in batches of variable size, Transportation sciences, 7, 85-99.

[4] Briere, G. and Chaudhry, M.L., 1989, Computational analysis of single server bulk-service queues, M/GY/1, Advance Application of Probability. 21, 207-225.

[5] Chaudhry, M.L. and Templeton, J.G.C.,1983, A First Course in Bulk Queues, Wiley, New York.

[6] Cohen, J.W., 1980, The Single Server Queue, 2nd edition, North-Holland, Amsterdam .

[7] Dhas, A.H.,1989, Markovian General Bulk Service Queueing Model, Ph.D. thesis, Dept. of Math, PSG College of Tech, India.

[8] Doshi, B.T., 1986, Queueing systems with vacations- A survey Queueing Systems 1, 29-66.

[9] Dshalalow, J.H. and Yellen., 1996, Bulk input queues with quorum and multiple vacations, Mathematical Problems in Engineering 2:2, 95-106.

[10] Downton, F.,1955, Waiting time in bulk service queues, Journal of Royal Statistical Society B17, 256-261.

[11] Fakinos, D., 1991, The relation between limiting queue size distributions at arrival and departure epochs in a bulk queue, Stochastic Processes. 37, 327-329.

[12] Grassmann, W.K. and Chaudhry M.L., 1982, A new method to solve steady state queueing equations, Naval Res. Logist. Quart. 29:3.

[13] Jaiswal, N.K., 1964, A bulk service queueing problem with variable capacity, Journal of Royal Statistical Society,B26, 143-148.

[14] Kambo, N.S. and Chaudhry, M.L., 1985, A single-server bulk-service queue with varying capacity and Erlang input, INFOR 23:2, 196-204.

[15] Medhi, J., 1975, Waiting time distribution in a Poisson queue with a general bulk service rule, Mgmt. Sci. 21:7, 777-782.

[16] Miller, R.G., 1959, A contribution to the theory of bulk queues, Journal of Royal Statistical Society, B21,320337.

[17] Neuts, M.F., 1967, A general class of bulk queues with Poisson input, Ann. Math. Stat. 38,757-770.

[18] Powell, W.B., 1987, Waiting time distribution for bulk arrival, bulk service queues with vehicle holding and cancellation strategies, Naval Res. Logist. 34, 207-227.

[19] Takagi, H., 1991, Queueing Analysis: A Foundation of Performance Evaluation, Vol. I, Vacation and Priority Systems, Part I, North-Holland. 